A N N A L E S Annales de Bretagne et des Pays de l'Ouest

\title{
Jean-Pierre Leguay, L'eau dans la ville au Moyen Âge
}

\section{Florian Mazel}

\section{(2) OpenEdition}

\section{Journals}

Édition électronique

URL : http://journals.openedition.org/abpo/1484

DOI : $10.4000 /$ abpo. 1484

ISBN : 978-2-7535-1489-8

ISSN : 2108-6443

Éditeur

Presses universitaires de Rennes

Édition imprimée

Date de publication : 20 mars 2003

Pagination : 167-168

ISBN : 978-2-86847-811-5

ISSN : 0399-0826

Référence électronique

Florian Mazel, « Jean-Pierre Leguay, L'eau dans la ville au Moyen Âge », Annales de Bretagne et des Pays de l'Ouest [En ligne], 110-1 | 2003, mis en ligne le 20 mars 2005, consulté le 21 septembre 2020. URL http://journals.openedition.org/abpo/1484 ; DOI : https://doi.org/10.4000/abpo.1484 
à un autre. Cette résidence au caractère palatial doit être interprétée dans le cadre de la défense contre les Bretons et a peut-être joué un rôle dans la réorganisation des pouvoirs à la fin des temps carolingiens quand s'affrontent pour la direction du comté du Maine les Rorgonides et les Hugonides. Finalement, sous la direction de Geoffroy de Mayenne se développera, au XI ${ }^{\mathrm{e}}$ siècle, une puissante baronnie qui saura jouer de sa position de frontière mais là l'historien entre dans un domaine certes à explorer mais un peu moins opaque.

On le voit l'intérêt suscité par ce remarquable volume est multiple et permet de progresser dans un domaine encore incertain pour une meilleure connaissance du milieu aristocratique.

Daniel PICHOT

Leguay, Jean-Pierre, L'eau dans la ville au Moyen Âge, Rennes, PUR, " Histoire ", 2002, $492 \mathrm{p}$.

Dans cet ouvrage de synthèse, l'auteur entend présenter la place et le rôle de l'eau dans les villes médiévales, du très haut Moyen Âge au début du Xvi siècle. Le propos s'organise en quatre grandes parties, d'abord chronologiques puis thématiques. Une première partie plonge ses racines dans le Bas-Empire pour se poursuivre jusqu'au XIII ' siècle et met l'accent sur le rôle de l'eau dans le choix du site et l'organisation topographique des cités. L'analyse du devenir variable des structures héritées de l'urbanisme romain retient particulièrement l'attention, de même que la réapparition chez les élites, aux XII ${ }^{\mathrm{e}}$-XIII ${ }^{\mathrm{e}}$ siècles, de préoccupations pour les œuvres d'intérêt public liées à la gestion de l'eau, qu'il s'agisse de problèmes d'approvisionnement ou d'évacuation. Une deuxième partie prolonge ce dernier propos, de manière plus précise, pour les $\mathrm{XIV}^{\mathrm{e}}$ et $\mathrm{XV}^{\mathrm{e}}$ siècles, une période qui bénéficie de sources plus abondantes, en rapport étroit avec l'accroissement des problèmes de pollution d'une part et avec l'essor d'une volonté et de moyens politiques (municipaux ou princiers) d'autre part. Les usages économiques de l'eau dans la ville constituent le sujet de la troisième partie, qui passe en revue les usages agricoles péri-urbains, les activités commerciales fluviales et les activités artisanales consommatrices d'eau (meunerie, teinturerie, tannerie, papeterie). Une dernière partie s'intéresse enfin à la place de l'eau dans ce qui relève, bien que l'auteur n'utilise pas l'expression, des "structures du quotidien ", qu'il s'agisse de la résidence ou des loisirs, des rites, des fêtes et des symboles, ou encore des accidents météorologiques et démographiques.

Comme le précise l'introduction, l'ouvrage ne s'intéresse qu'à la France, dans ses limites actuelles. La pauvreté des sources, le retard des recherches et la relative rareté de la bibliographie spécialisée, au regard des situations allemande et surtout italienne, pouvaient justifier une telle réduction du champ de l'étude, bien que cette réduction aurait dû apparaître dans le titre. On peut toutefois regretter que les analyses ne se prolongent pas, hormis de brèves allusions, par des comparaisons avec ces villes, italiennes en particulier, qui ont donné lieu à de nombreuses et brillantes études. Dans le domaine français, le panorama est très large même si, compte tenu des domaines de prédilection de l'auteur, on ne s'étonnera pas de voir la Bretagne, la Normandie et la Savoie privilégiées. Mais l'ensemble des autres régions de France sont envisagées et l'on dispose en particulier de développements substantiels pour 
la Flandre, Paris ou la Provence. L'équilibre est bien tenu aussi entre les capitales princières et grandes villes commerciales d'une part, et les très nombreuses petites villes qui constituent l'essentiel du réseau urbain de la France des $\mathrm{XII}^{\mathrm{e}}-\mathrm{XV} \mathrm{e}^{\mathrm{e}}$ siècles d'autre part. D'une manière générale, le propos est varié et abonde en exemples de toutes sortes, puisés dans une bibliographie fournie. Les actes de la pratique, les textes législatifs, la littérature, l'archéologie, l'iconographie sont tour à tour convoqués pour illustrer tel ou tel développement ponctuel. Figurent en outre dans l'ouvrage un certain nombre de textes utiles comme, par exemple, des règlements municipaux pour l'entretien des canaux ou des égouts, des extraits de comptes de fontainiers princiers ou encore la fameuse charte d'Henri II Plantagenêt au sujet des levées angevines de 1161. Sur le fond, plusieurs passages éveillent plus spécialement l'intérêt : l'aménagement des espaces marécageux, l'impact des équipements portuaires, l'implantation des moulins, les inondations et les réactions qu'elles suscitent. Un index thématique peut aider à retrouver les études de cas que la table des matières aurait pu laisser échapper.

Quoiqu'il faille considérer cet ouvrage comme une synthèse générale, une sorte de tableau, et non comme le résultat d'un travail de recherche, quelques critiques peuvent lui être adressées. Son usage potentiel comme instrument de travail est compromis par l'absence d'un index topographique et d'une table des illustrations et documents, comme par la médiocre qualité de nombreux plans de villes. Plusieurs d'entre eux sont en effet illisibles, que la qualité de l'impression soit médiocre (p. 37,62) ou que la taille des caractères soit trop petite (p. 78, 80, 179, 254). Certains sont même privés de leurs légendes (p. 51). D'autre part, ils ne concordent pas toujours avec le texte qui les commente : le plan d'Arles situé p. 17, censé illustrer le maintien du parcellaire en damier de la colonie romaine, ne comporte aucun tracé de rue ${ }^{1}$; le plan de Dinan, censé illustrer le choix d'un site de plateau, ne comporte ni courbe de niveau, ni tracé de la Rance (p. 49); les fontaines de Périgueux citées dans le commentaire p. 185 ne figurent pas sur le plan de la ville de la page précédente... Par ailleurs, l'ouvrage souffre dans l'ensemble de l'absence de véritable ligne directrice structurant le propos. Le champ du sujet n'est pas défini de manière problématique et l'eau fait en réalité figure de plus petit dénominateur commun de thèmes disparates, plus qu'elle ne vient soutenir une approche originale du système urbain, dans ses dimensions topographique, sociale, économique ou politique.

Florian MAZEL

Chagniot, Jean, Guerre et société à l'époque moderne, Paris, PUF, « Nouvelle Clio ", 2001,360 p.

La collection Nouvelle Clio n'a publié que de rares ouvrages à l'histoire militaire, dont fort peu sont consacrés à l'étude des relations entre guerre et société;

1. Au sujet d'Arles, on me permettra de rectifier ici quelques erreurs : la ville n'est pas située dans un estuaire mais à la tête d'un delta (contra, p. 331); son quartier le plus élevé se nomme la Hauture et non l'Auture, et le Vieux Bourg, premier des faubourgs de la cité, est dominé par la famille chevaleresque et non bourgeoise, des Porcelet (contra p. 17). 